\title{
Totally robotic versus totally laparoscopic surgery for rectal cancer
}

\author{
Bulent Gurbuz ${ }^{1}$, Serkan Zenger ${ }^{1}$, Emre Ozoran ${ }^{2}$, Emre Balik $^{2}$, Dursun Bugra ${ }^{1,2}$ \\ ${ }^{1}$ Department of General Surgery, VKF American Hospital, Istanbul, Turkey; ${ }^{2}$ Department of General Surgery, School of Medicine, Koc University, \\ Istanbul, Turkey \\ Correspondence to: Bulent Gurbuz. VKF American Hospital, Department of General Surgery, Guzelbahce ST, No. 20, Sisli, Istanbul, Turkey. \\ Email: drbulent_gurbuz@hotmail.com.
}

\begin{abstract}
Heald and Ryall introduced surgical techniques consisting of the complete removal of the mesorectum, which was named total mesorectal excision (TME) in the 1980s. The main outcome that could be achieved in the surgical treatment of rectal cancer is obtaining a good quality mesorectal excision besides enough harvested lymph nodes. TME reduces the risk of local recurrence and improves survival in patients with mid and low rectal disease. Many studies have indicated that minimally invasive surgery provides a faster postoperative recovery and a lower complication rate compared to conventional surgery. Ever since laparoscopic colectomy was done in 1991, its volume has increased over the last 30 years. In 2002 the first robotic-assisted colectomy article was published and in 2006 the first robotic-assisted TME article was published. After a learning curve for robotic surgery, it could be possible complete TME for oncological outcomes. Many studies have shown that there were no differences in terms of surgical and oncological outcomes between robotic and laparoscopic methods in rectal surgery. The aim of this video presentation study is to report the technical details of laparoscopic and robotic approaches for rectal cancer surgery and to state the similarities and differences between these two methods.
\end{abstract}

Keywords: Totally laparoscopic; totally robotic; rectal cancer

Received: 16 February 2020; Accepted: 07 September 2020; Published: 20 October 2020.

doi: 10.21037/ales-20-39

View this article at: http://dx.doi.org/10.21037/ales-20-39

\section{Introduction}

Rectal cancer surgery has seen a magical change from open to minimally invasive approach and has been revolutionized by minimally invasive surgery in the last two decades.

Heald and Ryall introduced surgical techniques consisting of the complete removal of the mesorectum, which was named total mesorectal excision (TME) in the 1980s (1,2). Being the rectal cancer gold standard TME has provided a low recurrence rate (3). The main outcome that could be achieved in the surgical treatment of rectal cancer is obtaining a good quality mesorectal excision besides enough harvested lymph nodes. (4). In our series of laparoscopic and robotic TME mean harvested lymph nodes was 27; without any significant difference between the two groups.

The use of minimally invasive surgery in rectal cancer had been controversial (5). Esen et al. reported that robotic and laparoscopic surgery for the treatment of rectal cancer could be utilized safely. Both techniques provide good specimen quality and are associated with adequate morbidity both in the operative and postoperative period. No difference in the number of harvested lymph nodes was seen between robotic and laparoscopic surgery for rectal cancer (4). All procedures performed in studies involving human participants were in accordance with the ethical standards of the institutional and/or national research committee(s) and with the Helsinki Declaration (as revised in 2013). Written informed consent was obtained from the patient. 


\section{Preoperative preparations}

Before surgery, the diverting ileostomy sides are routinely marked with permanent markers by the stoma-therapy nurse. Generally, the total number of four possible sites are marked. We routinely perform a diverting ileostomy in patients who have undergone neoadjuvant radiotherapy and/or when the tumor is located in the mid-distal rectum. Mechanical bowel preparation is administered the day before the operation to all of the patients except in patients with an obstructing lesion. Low molecular weight heparin for venous thromboembolism prophylaxis is started 12 hours before the surgery. In the operating room, a central venous catheter and thoracic epidural catheters are inserted by the anesthesiologist. Under general anesthesia and epidural analgesia, Allen stirrups are utilized and a modified lithotomy position is established. A urinary catheter is inserted through the urethra for the emptying of the urinary bladder. During the surgery and in the postoperative period urinary output is monitored with this catheter. The patient's legs are placed in Allen stirrups, slightly bent and abducted.

To prevent patient slipping during surgery, supports are used bilaterally to the upper arm on the lateral sides ad shoulders; since the patient position is variable through the various steps of the surgical procedure (reverse and steep Trendelenburg with right and left tilting). The patient is prepped starting from the perineum. After the preparation of the perineum abdominal preparation starts from the xiphoid process until the pubic symphysis from superior to inferior; between the posterior axillary lines laterally.

The similar procedures of laparoscopic and robotic techniques are provided in the common text while the differences are specifically highlighted.

\section{Operative technique (Video 1)}

\section{Patient positioning and trocar placement for laparoscopic surgery}

The surgeon and assistance (camera) stay on the patient's right side. We routinely induce pneumoperitoneum with a Veress needle which is placed about $2 \mathrm{~cm}$ superior to the umbilicus on the midline in patients who did not have a previous abdominal surgery. The Veress needle is placed in the left upper quadrant in patients who had undergone an abdominal procedure previously. The insufflation pressure is set to $12 \mathrm{mmHg}$. A $10 \mathrm{~mm}$ (camera) trocar is trusted from the Veress needle site. A telescope is placed through

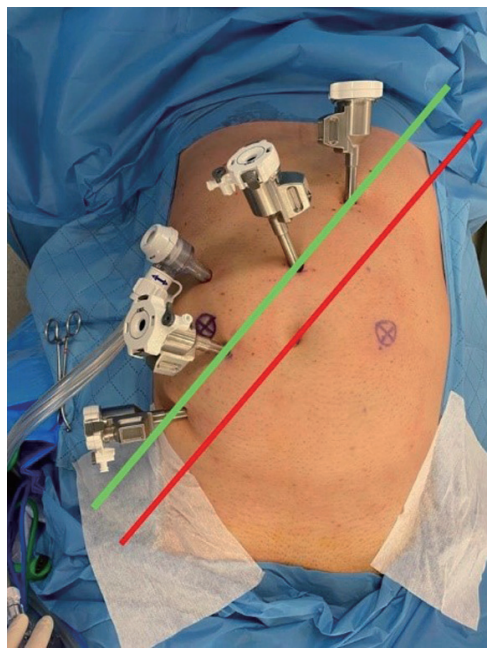

Figure 1 Trocar placement site for robotic rectal surgery.

this trocar into the abdomen. Two $5 \mathrm{~mm}$ trocars are placed bilaterally 6-7 $\mathrm{cm}$ from the umbilicus. The one on the left will be used by the assistant and the one on the right will be used by the left hand of the surgeon. For the right hand of the surgeon, a $12 \mathrm{~mm}$ trocar is placed in the right iliac fossa.

The supposed placement of the trocars allows for the proper and ergonomic dissection of the left colon, splenic flexure in addition to an optimal dissection of the pelvis to achieve mesorectal excision. After the placement of the trocar, the patient is put in steep Trendelenburg position and tilted to the right side. Thus, the small bowel moves to the right upper quadrant providing optimal exposure.

\section{Patient positioning and trocar placement for robotic surgery}

A line is drawn from the subcostal margin (at the level of the 8th rib junction) to the approximate location of the right femoral head "costofemoral line" (red line in Figure 1). A-line $4 \mathrm{~cm}$ parallel to the costofemoral line towards the hepatic flexure "offset line" (green line Figure 1) is drawn. Initial endoscope port will be put at the crossing between the green line with the midline, thus a Veress needle is thrust into the abdominal cavity $2-3 \mathrm{~cm}$ superior to the umbilicus along the midline. After carbon dioxide insufflation at $12 \mathrm{mmHg}$, a trocar with a diameter of $12 \mathrm{~mm}$ is thrust and the exploration is performed using the telescope of the Da Vinci Xi system.

"Port 1" (8 $\mathrm{mm}$ robotic port) is put superior to the endoscope port on the green line. Ports 3 and 4 are placed 
on the dashed line inferior to the endoscope port. Airseal trocar (another $8 \mathrm{~mm}$ special trocar) is put for the assistant, on the lateral side and it is triangulated between $3 \mathrm{rd}$ and 4th ports. On the system "LOWER ABDOMINAL" is selected for anatomy, "PATIENT LEFT" is selected for the approach. The operating table is prepared at 10 degrees Trendelenburg, rolled to the right side at 10 degrees, and the height is adjusted to the maximum lowest level.

The patient cart is deployed for docking (DEPLOY). The patient cart is driven so that the green laser is positioned on the initial endoscope port. Adequate clearance must be ensured between the patient and the da Vinci arms. Then Arm 2 is docked with the endoscope port. "FLEX" is adjusted between " $\mathrm{L}$ " and "E". The scope is pointed to the sigmoid colon and "TARGETING" is performed. The other arms are docked.

\section{Vascular dissection, mobilization of the sigmoid and the left colon}

Exploration of the abdominal cavity is the primary step of the operation. In our clinic, we prefer medial to lateral dissection. Visualization of the inferior mesenteric artery (IMA) is the first step in sigmoid colon dissection. At the promontorium level, the peritoneum is cut open on the right side. The dissection continues from this level until the pedicle of the IMA. The dissection continues below the mesentery of the descending colon. To save the left ureter and hypogastric nerve plexus, the surgeon should adhere to the right dissection plane. The IMA must be dissected close to its aortic root to achieve high ligation for better oncological results. The surgeon should be careful to preserve the superior hypogastric nerve plexus while the IMA is dissected. During this dissection, the surgeon should adhere to sticking in the right plane in front of the Gerota fascia also called the embryogenic plane to prevent injury to the left ureter. Although it is not always obligatory to see the ureter. After the IMA dissected, clips are placed and it is incised close to its aortic root (high ligation). Instead of clipping, it could also be ligated with energy devices.

The dissection (medial to lateral) continues superiorly. The inferior mesenteric vein is dissected on its course and either clipped or ligated near the inferior border of the pancreas.

The left and sigmoid colon are dissected from medial to lateral in the avascular plane of Toldt, thus retroperitoneal and intraperitoneal structures pertaining to embryologically different structures. The lateral end of the abdominal wall is the last stop in the medial to lateral dissection of the retroperitoneum. Toldt's fascia is found and defined, the left mesocolon is dissected from the retroperitoneum. After the medial dissection is completed, the lateral to medial dissection starts.

\section{Lateral to medial dissection}

The lateral to medial dissection initiates, with the medial retraction of the left colon. The attachments on the lateral sides of the sigmoid and the left colon are incised for the complete mobilization of the colon. Then lateral to medial dissection continues superiorly. An inferior approach is utilized to incise the transverse mesocolon from the inferior border of the pancreas. The complete mobilization of the splenic flexure is performed by the division of the gastrocolic ligament and the lateral attachment to the peritoneum. We usually mobilize splenic flexure for rectal cancer surgery.

In our clinic, we use single docking for the mobilization of the splenic flexure when performing robotic surgery. In laparoscopic surgery, we use a second monitor, the trocar for the telescope is unchanged although the surgeon uses the assistant left trocar.

Following complete mobilization of the splenic flexure, the retroperitoneal structures become apparent. Renal vein left kidney, aorta and gonadal vessels can be seen. Mobilization of the rectum is the latter step.

\section{Rectum mobilization}

The dissection is initiated from the posterior part of the rectum, then continued on the lateral sides and finished on the anterior part. When laparoscopic energy devices are used these must be utilized by the assistant. The dissection continues on the right and left side and finalized on the anterior aspect.

Seminal vesicles and vagina are the potentially damaged structures during the anterior dissection. The insertion of a vaginal probe or digital vaginal examination by the assistant could help the surgeon. If an injury is detected it allows for prompt repair.

On the sides, shifting planes could end up injuring the ureters. On the anterior aspect; in the female patient, the uterus could sometimes block the way of proper dissection. It could be retracted by a probe or could be stitched up the anterior abdominal wall for retraction.

Obtaining a complete circumferential resection margin is 
one of the paramount outcomes of rectal surgery. Dissection through the right surgical planes is the leading factor in achieving a complete circumferential resection margin.

Some factors are challenging for the colorectal surgeon performing a rectal resection, especially during pelvic dissection. These are patients with a body mass index of more than 30, patients with a narrow pelvis, patients with bulky, large tumors, patients who underwent radiotherapy and/or surgery for other pelvic malignancies. A wise colorectal surgeon should always consider conversion to open surgery when needed.

After the dissection plane arrives at the levator muscles, by then full mobilization of the rectum is achieved, the rectum is lifted superiorly. With a rectal digital examination, the level of transection is evaluated. Through the $12 \mathrm{~mm}$ trocar on the right iliac fossa, an endoscopic linear stapler is placed. With the linear stapler rectum is closed then it is incised a few centimeters superior of the dentate line. During this procedure, an assistant may provide pressure to the perineum externally. The preparation of the distal rectum should be adequate thus no more than two cartridges would be needed for transection of the rectum. The proper preparation of the rectum is needed to avoid an increased risk of anastomotic leakage. After the transection, the transected rectum is caught with one grasper

\section{Intracorporeal mesocolic transection}

An area for proximal transection on the descending colon is determined. The mesentery of the colon is ligated and incised with energy devices until the colon is reached. Intravenous indocyanine green (ICG) is used for fluorescence angiography. Following the injections, vascular structures become enhancing visible aiding us to confirm an area with good vascular perfusion for the selection of the transection point. A clip is placed on this point to determine this area when the colon is taken out from the Pfannenstiel incision.

\section{Specimen extraction}

A $4-5 \mathrm{~cm}$ vertical suprapubic incision (Pfannenstiel) is preferred in our clinic. A small plastic wound protector is placed. The colon is taken out through the wound protector, the specimen is divided from the left colon and it is brought for the frozen examination of the distal surgical margin. The anvil of the circular stapler $(28$ or $31 \mathrm{~mm}$ ) is introduced to the proximal end of the colon and a 2-0 polypropylene is used to achieve a purse string. The colon placed back in the abdominal cavity. The wound protector is closed and insufflation is initiated for pneumoperitoneum.

\section{Colorectal anastomosis}

Colo-anal anastomosis with a circular stapler is preferred. The stapler is placed through the anal canal. The pin is attached to the anvil while the actions are monitored on the screen. The stapler is fired and end-to-end anastomosis is performed. The stapler is taken out of the anal canal, the doughnuts are checked for completeness.

Air-leak test is undertaken. When the air-leak test is positive the anastomosis should be checked. When a defect is found the options could be placing sutures to close the defect, performing re-anastomosis. When a defect is not visible intraoperative endoscopy could be utilized to check the anastomosis endoluminal.

A drain (closed suction) is placed into the pelvis from the left lower trocar. In robotic surgery, the drain is placed through the most inferior $8 \mathrm{~mm}$ port site. In our practice, the drain is wrapped around the neo-rectum, although it could be placed without wrapping directly into the pelvis.

An intestinal loop is chosen for diverting ileostomy about $40 \mathrm{~cm}$ proximal to the caecum and marked with stapler for determining the afferent and efferent loops. It is taken out from the preoperatively marked stoma site.

After ending the pneumoperitoneum, the Pfannenstiel incision is closed with loop PDS. The fascial defects in the port sites larger than $8 \mathrm{~mm}$ are repaired with $2 / 0$ vicryl sutures. The incisions are sutured primarily. The intestinal loop is matured to loop ileostomy.

\section{Postoperative care}

The patient is followed either in the intensive care unit or the surgical ward after the surgery according to the patients' comorbidities. Nasogastric tube is not used routinely. The patient is given a few glasses of water after the surgery, and soft regime is ordered in the first postoperative day. Antibiotics are not used routinely, except prophylaxis if there is no leakage. The patient is mobilized and respiratory exercises are started a few hours after the surgery.

\section{Acknowledgments}

Funding: None. 


\section{Footnote}

Conflicts of Interest: All authors have completed the ICMJE uniform disclosure form (available at http://dx.doi. org/10.21037/ales-20-39). The authors have no conflicts of interest to declare.

Ethical Statement: The authors are accountable for all aspects of the work in ensuring that questions related to the accuracy or integrity of any part of the work are appropriately investigated and resolved. All procedures performed in studies involving human participants were in accordance with the ethical standards of the institutional and/or national research committee(s) and with the Helsinki Declaration (as revised in 2013). Written informed consent was obtained from the patient.

Open Access Statement: This is an Open Access article distributed in accordance with the Creative Commons Attribution-NonCommercial-NoDerivs 4.0 International License (CC BY-NC-ND 4.0), which permits the noncommercial replication and distribution of the article with

doi: 10.21037/ales-20-39

Cite this article as: Gurbuz B, Zenger S, Ozoran E, Balik E, Bugra D. Totally robotic versus totally laparoscopic surgery for rectal cancer. Ann Laparosc Endosc Surg 2020;5:38 . the strict proviso that no changes or edits are made and the original work is properly cited (including links to both the formal publication through the relevant DOI and the license). See: https://creativecommons.org/licenses/by-nc-nd/4.0/.

\section{References}

1. Heald RJ. A new approach to rectal cancer. Br J Hosp Med 1979;22:277-81.

2. Heald RJ, Ryall RD. Recurrence and survival after total mesorectal excision for rectal cancer. Lancet 1986;1:1479-82.

3. Dahlberg M, Påhlman L, Bergström R, et al. Improved survival in patients with rectal cancer: a population-based register study. Br J Surg 1998;85:515-20.

4. Esen E, Aytac E, Ağcaoğlu O, et al. Totally Robotic Versus Totally Laparoscopic Surgery for Rectal Cancer. Surg Laparosc Endosc Percutan Tech 2018;28:245-9.

5. Abbas SK, Yelika SB, You K, et al. Rectal cancer should not be resected laparoscopically: the rationale and the data. Tech Coloproctol 2017;21:237-40. 